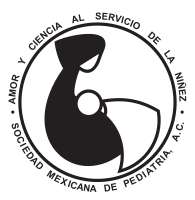

\title{
Marcapasos endocárdico en un prematuro con bloqueo AV y situs inversus total
}

\author{
Yeiscimin Sánchez-Escobedo, ${ }^{\ddagger * *}$ Carlos De la Fuente-Macip, ${ }^{\ddagger}$ Heberto Aquino-Bruno, ${ }^{\ddagger}$ \\ Mariana Lourdes Añas-Méndez, ${ }^{\ddagger}$ Karla Ivette Bozada-Nolasco, ${ }^{\ddagger}$ \\ Arturo Enríquez-Silverio, ${ }^{\ddagger}$ Ulises Rojel-Martínez ${ }^{\ddagger}$ \\ ¥ Laboratorio de Electrofisiología y Estimulación Cardiaca, Unidad de Hemodinamia, Servicio de Cardiología, \\ Complejo Médico Sur. Secretaría de Salud del Estado de Puebla. Puebla, México.
}

\begin{abstract}
RESUMEN
Objetivo: Se presenta el caso de un recién nacido prematuro con bloqueo auriculoventricular congénito (BAVC) y con situs inversus total (SIT), a quien se le colocó un marcapasos unicameral por vía endocárdica. Caso clínico: Femenino de dos días de vida extrauterina, de 35 semanas de gestación y bajo peso al nacimiento. Un electrocardiograma de 12 derivaciones mostró una frecuencia ventricular de 68 por minuto, ondas $P$ negativas en DI y aVL y positiva en aVR, y bloqueo auriculoventricular 2 $x 1$. El análisis secuencial segmentario concluyó situs inversus concordante y una tomografía axial computarizada toracoabdominal confirmó SIT. Debido a que en las primeras 48 horas se observaron datos de bajo gasto cardiaco, y después de descartar causas de BAVC, se decidió colocar un marcapasos unicameral por vía endocárdica. La paciente evolucionó sin complicaciones. Conclusión: EI SIT es una condición que puede asociarse a BAVC; la implantación de marcapasos, inclusive en prematuros, puede mejorar el pronóstico de estos pacientes.
\end{abstract}

Palabras clave: Bloqueo auriculoventricular congénito, marcapasos definitivo, situs inversus total, recién nacido.

\section{INTRODUCCIÓN}

El bloqueo auriculoventricular congénito (BAVC) ocurre en uno de cada 25,000 recién nacidos $(\mathrm{RN}){ }^{1}$ La causa más frecuente en corazones estructuralmente normales son las enfermedades autoinmunes, debido al paso transplacentario de autoanticuerpos anti-Ro/SSA y anti-La/SSB maternos que producen alteraciones en la célula cardiaca. Las cardiopatías estructurales representan la segunda causa de BAVC. ${ }^{2}$

\begin{abstract}
Objective: We report the case of a premature newborn with congenital atrioventricular block (CAVB) and with situs inversus totalis (SIT), in whom a single-chamber endocardial pacemaker was implanted. Clinical case: Two-day-old female who was born at 35 weeks gestation and with low birth-weight. A 12-lead electrocardiogram showed a ventricular rate of $68 \mathrm{bpm}$, negative $P$ waves in D1 and $\mathrm{aVL}$ and positive in $\mathrm{aVR}$, with atrioventricular block $2 \times 1$. Sequential segmental analysis determined situs inversus, while a thoracic-abdominal CT scan confirmed SIT. Because data on low cardiac output were observed in the first 48 hours, and after ruling out causes of BCVB, it was decided to implant a single-chamber pacemaker endocardially. The patient evolved without complications. Conclusion: SIT is a condition that can be associated with BCVB; pacemaker implantation, even in premature infants, can improve the prognosis for these patients.
\end{abstract}

Keywords: Congenital atrioventricular block, definitive

pacemaker, situs inversus total, newborn.

\footnotetext{
* Correspondencia: YSE, yeiscimin@gmail.com

Conflicto de intereses: Los autores declaran que no tienen. Citar como: Sánchez-Escobedo Y, De la Fuente-Macip C, Aquino-Bruno H, Añas-Méndez ML, Bozada-Nolasco KI, EnríquezSilverio $A$ et al. Marcapasos endocárdico en un prematuro con bloqueo AV y situs inversus total. Rev Mex Pediatr 2020; 87(2):7478. doi: $10.35366 / 94172$

[Endocardial pacemaker in a premature infant with AV block and situs inversus totalis]
} 
Dentro de las alteraciones anatómicas existen los defectos de lateralidad donde se pierde el eje embrionario de desarrollo, dando lugar a la inversión de la simetría del corazón, lo cual se denomina situs inversus (SI). ${ }^{3}$ Esta es una entidad rara caracterizada por imagen en espejo de vísceras torácicas, estimándose que tiene una prevalencia de 1 a 2 en $10,000 \mathrm{RN}$ vivos. ${ }^{4} \mathrm{El}$ BAVC en fetos con síndrome de heterotaxia o isomerismo se comporta como un factor de riesgo de muerte en el periodo perinatal, ${ }^{5}$ en estos casos la tasa de mortalidad es superior a $50 \%$ en los primeros seis meses de vida y de hasta $67 \%$ a los dos años. ${ }^{6}$ El tratamiento de elección es la colocación de un marcapasos definitivo. ${ }^{7}$

Dado que hay pocos reportes en pacientes pediátricos, se presenta el caso de un RN prematuro con SI y BAVC sintomático a quien se le implantó un marcapasos unicameral endocárdico como tratamiento definitivo.

\section{PRESENTACIÓN DEL CASO}

Femenino de dos días de vida extrauterina producto pretérmino de la primera gestación. La madre tenía 38 años y con diabetes mellitus tipo 1, en tratamiento con insulina.

A las 35 semanas de gestación un ultrasonido reportó bradicardia de 90 latidos por minuto (lpm), por lo que se decidió realizar cesárea, obteniéndose producto vivo hiporreactivo con cianosis generalizada y dificultad respiratoria. Su peso fue de 2,660 gramos, talla de 49 centímetros, Apgar de 6/7 puntos, Silverman de 3 y saturación de $88 \%$; se realizaron maniobras básicas de reanimación con adecuada respuesta, sin embargo, persistió con bradicardia.
Se realizó un electrocardiograma de 12 derivaciones en el que se observó una frecuencia ventricular de 68 lpm, ondas P negativas en DI y aVL, es positiva en aVR; eje eléctrico a 150 grados y bloqueo auriculoventricular de 2 x 1 y un intervalo QTm de 0.36 segundos y QTc de 0.38 segundos (Figura 1). Por ecocardiograma, dado por análisis secuencial segmentario se concluyó: situs inversus del segmento auricular morfológicamente derecho con unión auriculoventricular concordante de asa ventricular D (derecha) y segmento arterial concordante; además se identificó una comunicación interventricular perimembranosa de $3 \mathrm{~mm}$. La radiografía de tórax mostró dextrocardia (Figura 2); mientras que la tomografía toracoabdominal simple corroboró el SI.

A las 48 horas, continuaba con bradicardia y hemodinámicamente inestable, pese al tratamiento médico, por lo que se decidió la colocación de marcapasos definitivo al tercer día. Por supuesto, se descartaron causas transitorias de BAVC, como trastornos electrolíticos o procesos infecciosos.

La instalación del marcapasos se realizó bajo anestesia general e intubación endotraqueal. La punción de la vena subclavia derecha fue guiada por fluoroscopía; se utilizaron dilatadores 4, 5 y $6 \mathrm{~F}$ de forma progresiva $\mathrm{y}$, finalmente, con introductor $7 \mathrm{~F}$ se introdujo un electrodo bipolar de fijación activa Fineline ${ }^{\circledR}$ Boston Scientific ${ }^{\circledR}$ de $52 \mathrm{~cm}$, colocándolo a nivel medioseptal, se obtuvo una onda $\mathrm{R}$ de $18 \mathrm{mV}$, una impedancia de $876 \mathrm{Ohms}$ y un umbral de captura de $1.4 \mathrm{~V}(0.4 \mathrm{~ms})$. Se utilizó un generador de impulsos Microny ${ }^{\circledR}$, Abbott $^{\circledR}$ colocándose a nivel submuscular (Figura 3). No hubo complicaciones durante el procedimiento.

Días después se descartaron otras posibles causas de bloqueo mediante tamiz neonatal y perfil autoinmune.

Figura 1:

Electrocardiograma de 12 derivaciones, ondas $\mathrm{P}$ negativas en DI y aVL, positiva en aVR, eje eléctrico desviado a la derecha; bloqueo auriculoventricular de $2 \times 1$ y un intervalo QTm de 0.36 segundos y QTc de 0.38 segundos.

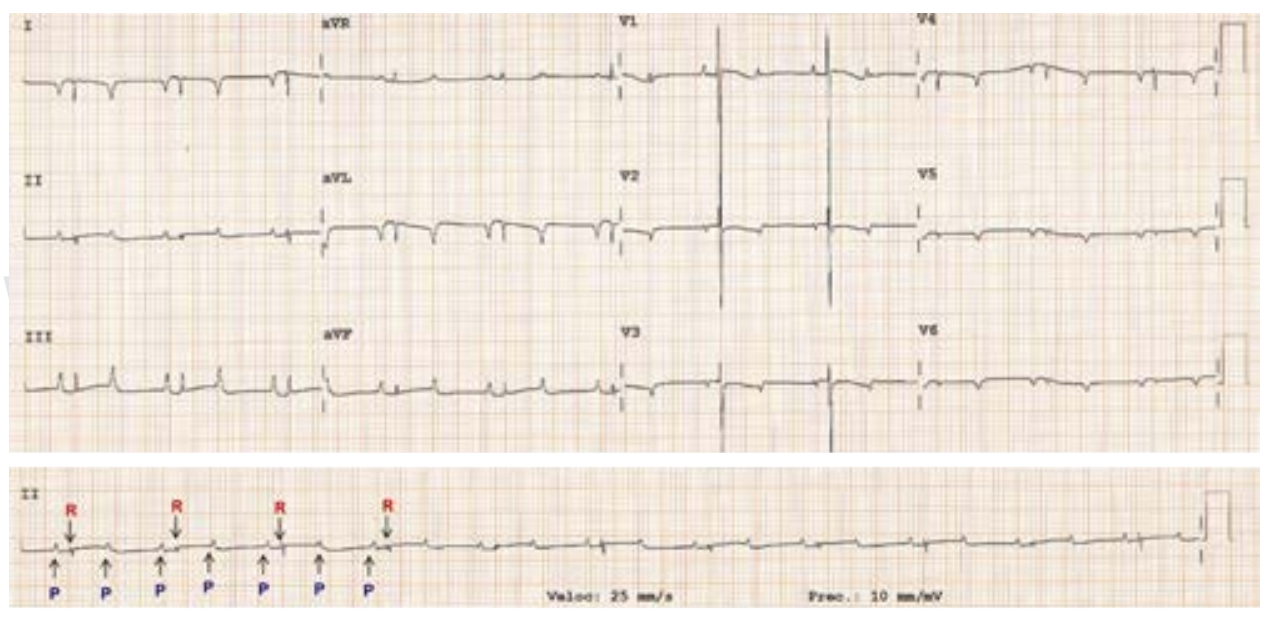




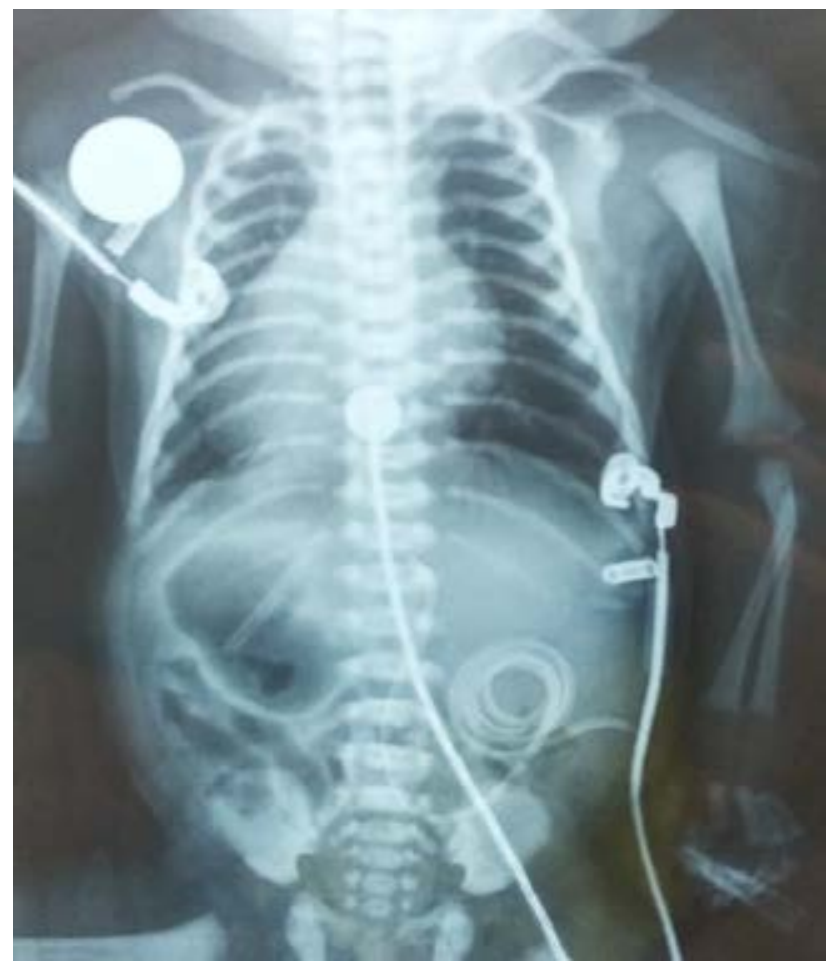

Figura 2: Radiografía anteroposterior al primer día de nacimiento. Se observa situs inversus total.

A los dos meses de seguimiento la paciente se encontraba asintomática desde el punto de vista cardiovascular, manteniendo el marcapasos funcionando apropiadamente (Figura 4), observando aumento de peso (3,500 g).

\section{DISCUSIÓN}

El BAVC puede asociarse a enfermedades maternas, tales como metabólicas, medicamentos o autoinmunes, siendo esta última la causa más frecuente. También se deben considerar las anomalías cardiacas anatómicas, las cuales llegan a alterar la conducción entre aurículas y ventrículos, lo cual se presenta entre 14 y $42 \%$ de los casos. ${ }^{8}$ Dentro de los defectos estructurales cardiacos congénitos las malformaciones complejas se llegan a presentar como un síndrome de heterotaxia (SH) en $0.8 \%,{ }^{9}$ en estos casos se ha reportado taquicardia supraventricular, BAV y enfermedad del nodo.

En un estudio retrospectivo que evaluó la supervivencia de 35 lactantes, el SH se asoció a algún tipo de arritmia en $83 \%$ durante un seguimiento de 65 meses, falleciendo $34 \%$ de los pacientes. Otro estudio de 40 niños con SH la prevalencia de bloqueo AV fue de $19 \% .{ }^{10}$ Escobar-Díaz y colegas ${ }^{11}$ reportaron que en 154 fetos con SH y arritmias $24 \%$ tenía BAVC, $79 \%$ sobrevivió al nacimiento y sólo 8\% fue tratado con marcapasos definitivo, destacando que fallecieron los pacientes en quienes se difirió el implante de marcapasos.

La implantación de marcapasos en la población pediátrica representa menos de $1 \%$ del total de implantes. ${ }^{12}$ La técnica de implante transvenoso de electrodos de marcapasos en pacientes pediátricos (menores de 12 meses y con peso bajo) ha sido poco documentada. Vos y colaboradores ${ }^{13}$ reportaron una serie de siete casos en lactantes menores de $10 \mathrm{~kg}$ a quienes se trató con implante transvenoso unicameral cuya indicación más frecuente fue el BAV completo en $53.7 \%$. La edad para implantar el marcapasos fue de dos días a 14 meses, y el promedio de peso al procedimiento fue de $3.5 \mathrm{~kg}$. En el seguimiento a 14 años se observó que $85 \%$ requirió reintervención para recambio a marcapasos bicameral, aproximadamente a los nueve años de edad.

En estudios prospectivos se han reportado complicaciones tempranas por la implantación del marcapasos, tales como oclusión de la vena subclavia (lo cual parece
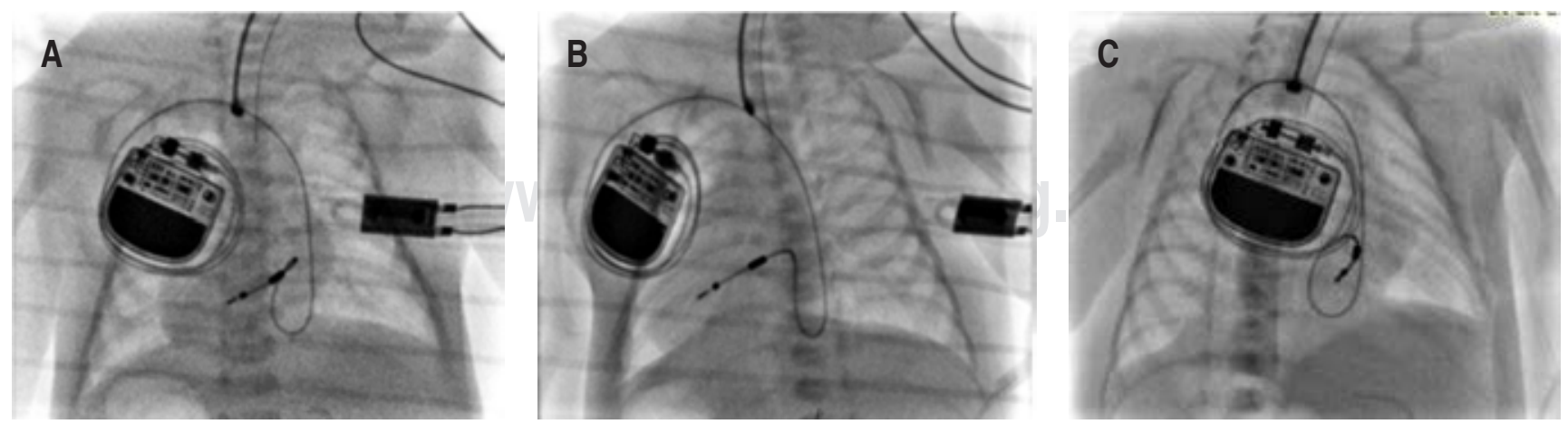

Figura 3: Proyecciones de fluoroscopía después del implante. A) Proyección AP. B) Proyección izquierda. C) Proyección derecha. 


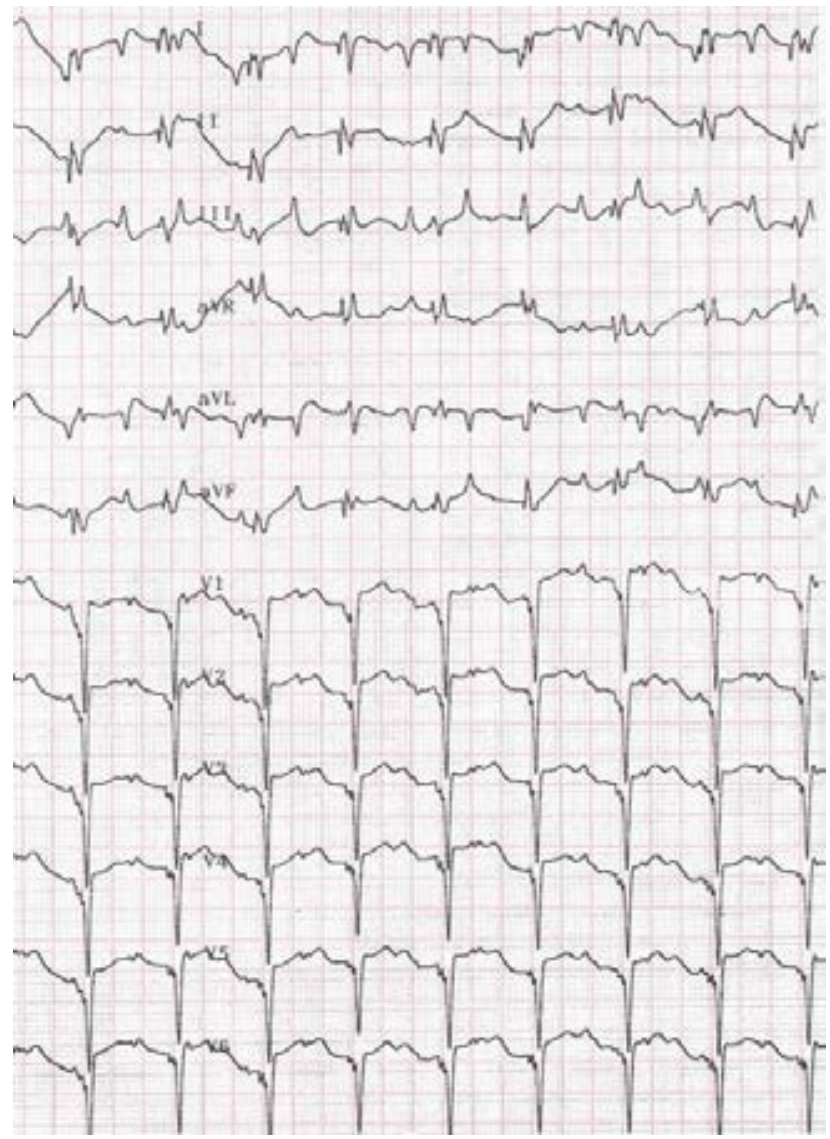

Figura 4: Electrocardiograma de 12 derivaciones, ritmo sinusal dependiente de marcapasos con frecuencia cardiaca programada a 100 latidos por minuto.

ser inversamente proporcional al peso), pero no se ha descrito colapso completo del acceso venoso. ${ }^{14} \mathrm{~A}$ largo plazo se ha visto que la complicación más frecuente es el estiramiento del electrodo. Nakamoto y colegas ${ }^{15}$ describieron a un paciente en quien se decidió exceder la porción endovascular del cable $(8 \mathrm{~mm})$, con el fin de evitar dicha eventualidad. No obstante, se debe considerar que es una reintervención.

Reportes de casos aislados argumentan que la técnica endocárdica tiene mayores ventajas que la epicárdica. Aunque los electrodos epicárdicos siguen siendo una opción importante en la estimulación de los pacientes pediátricos, es conocido que tienen diversos inconvenientes, como estar diseñados para pacientes adultos, la necesidad de una toracotomía, problemas de sensado y captura (en promedio consumen seis veces más energía que los endocárdicos) y menor longevidad. Por otro lado, la estimulación endocárdica tiende a te- ner un mejor umbral (aurícula y ventrículo) y un mejor acceso a los sitios de estimulación en comparación a la técnica abierta. ${ }^{14,15}$

En niños con peso menor a $10 \mathrm{~kg}$, los sistemas de estimulación pueden ser endo- o epicárdicos. Silvetti y colaboradore ${ }^{16}$ reportaron 287 pacientes con marcapasos, de los cuales $40.7 \%$ tenía sistema endocárdico; el análisis mostró un riesgo mayor de falla en los pacientes con implantes epicárdicos comparado con quienes se implantó de forma endocárdica (50\% versus 13\%), lo cual fue estadísticamente significativo ( $\mathrm{p}<0.0001)$. Distintos autores en los últimos 20 años proponen como mejor opción la estimulación endocárdica; sin embargo, aún no hay estudios concluyentes, por lo que es necesario tener en cuenta las características particulares de cada paciente. ${ }^{17,18}$ Lo anterior, teniendo en cuenta que el diseño de electrodos endocárdicos con menor diámetro y longitud, así como generadores de menor peso y tamaño, facilitan la técnica de implante endocárdico.

\section{REFERENCIAS}

1. Cortés-Ramírez RM, Cortés de la-Torre JM, Cortés de la-Torre R, Reyes-Méndez BJ, Salazar-de Santiago RA, Carrillo Aguilar SI et al. Bloqueo AV completo congénito. Revisión y presentación de un caso. Rev Mex Cardiol. 2013; 24(3): 144-146.

2. Li X, Huang X, Lu H. Two case reports of neonatal autoantibodyassociated congenital heart block. Medicine (Baltimore). 2018; 97(45): e13185.

3. Icardo JM, García Rincón JM, Ros MA. Congenital heart disease, heterotaxia and laterality. Rev Esp Cardiol. 2002; 55(9): 962-974.

4. Urquia-Osorio HO, Discua-Flores LJ, Valenzuela-Castillo R. Situs inversus totalis y cardiopatía congénita: diagnóstico casual en un lactante con neumonía. CIMEL. 2010; 15(2): 85-88.

5. Taketazu M, Lougheed J, Yoo SJ, Lim JS, Hornberger LK. Spectrum of cardiovascular disease, accuracy of diagnosis, and outcome in fetal heterotaxy syndrome. Am J Cardiol. 2006; 97(5): 720-724.

6. Aziz PF, Tanel RE, Zelster IJ, Pass RH, Wieand TS, Vetter VL et al. Congenital long QT syndrome and 2:1 atrioventricular block: An optimistic outcome in the current era. Heart Rhythm. 2010; 7(6): 781-785. doi: 10.1016/j.hrthm.2010.02.035.

7. Baruteau AE, Pass RH, Thambo JB, Behaghel A, Le Pennec S, Perdreau E et al. Congenital and childhood atrioventricular blocks: pathophysiology and contemporary management. Eur J Pediatr. 2016; 175(9): 1235-1248. doi: 10.1007/s00431-016-2748-0.

8. Brito-Zerón P, Izmirly PM, Ramos-Casals M, Buyon JP, Khamashta MA. The clinical spectrum of autoimmune congenital heart block. Nat Rev Rheumatol. 2015; 11(5): 301-312. doi: 10.1038/ nrrheum.2015.29.

9. McGovern E, Kelleher E, Potts JE, O'Brien J, Walsh K, Nolke L et al. Predictors of poor outcome among children with heterotaxy syndrome: a retrospective review. Open Heart. 2016; 3: e000328. doi: 10.1136/openhrt-2015-000328.

10. Ozawa Y, Asakai H, Shiraga K, Shindo T, Hirata Y, Hirata Y et al. Cardiac rhythm disturbances in heterotaxy syndrome. Pediatr Cardiol. 2019; 40(5): 909-913. doi: 10.1007/s00246-019-02087-2.

11. Escobar-Diaz MC, Tworetzky W, Friedman K, Lafranchi T, FynnThompson F, Alexander ME et al. Perinatal outcome in fetuses with 
heterotaxy syndrome and atrioventricular block or bradycardia. Pediatr Cardiol. 2014; 35(6): 906-913. doi: 10.1007/s00246-014-0874-x.

12. Cabrera OM, Alemán FAA, Benítez RDB. Implantación de marcapaso endocavitario en un niño de 2 años por fallo del sistema epicárdico. Rev Cubana Cardiol Cir Cardiovasc. 2013; 19(2): 62-65.

13. Vos LM, Kammeraad JAE, Freund MW, Blank AC, Breur JMPJ. Long-term outcome of transvenous pacemaker implantation in infants: a retrospective cohort study. Europace. 2017; 19(4): 581587. doi: 10.1093/europace/euw031.

14. Konta L, Chubb MH, Bostock J, Rogers J, Rosenthal E. Twentyseven years experience with transvenous pacemaker implantation in children weighing < $10 \mathrm{~kg}$. Circ Arrhythm Electrophysiol. 2016; 9: e003422. doi: 10.1161/CIRCEP.115.003422.

15. Nakamoto S, Fujii K, NIshino T, Satus T, Ogawa T, Kaneda T et al. Intravenous pacemaker lead implantation for a pediatric patient:
A 16- year follow- up study. Journal of Arrhythmia. 2013; 29(5): 287-290.

16. Silvetti MS, Drago F, Di-Carlo D, Placidi S, Brancaccio G, Carotti A. Cardiac pacing in paediatric patients with congenital heart defects: transvenous or epicardial? EP Europace. 2013; 15 (9): 1280-1286. doi: 10.1093/europance/eut029.

17. Haponiuk I, Chojnicki M, Szofer-Sendrowska A, Juscinski J, Steffens M, Jaworski R et al. "Planned" permanent pacemaker implantation in one-day-old newborn after prenatal diagnosis of congenital complete atrioventricular heart block. Kardiochir Torakochirurgia Pol. 2014; 11(1): 76-78. doi: 10.5114/ kitp.2014.41937.

18. Wilhelm BJ, Thone M, El-Scheich T, Livert D, Angelico R, Osswald B. Complications and risk assessment of 25 years in pediatric pacing. Ann Thorac Surg. 2015; 100: 147-153. doi: https://doi. org/10.1016/j.athoracsur.2014.12.098. 